\title{
IUFOST2006/1224 Interactions between pectin and cellulose
}

\author{
A. Zykwinska ${ }^{a}$, M. Ralet ${ }^{\mathrm{b}}$, C. Garnier ${ }^{\mathrm{c}}$ and J.-F. Thibault ${ }^{\mathrm{d}}$ \\ ${ }^{a}$ INRA - Research Unit : Biopolymers, Interactions, Assemblies, rue de la Géraudière, 44316 Nantes, France \\ bINRA Research Unit : Biopolymers, Interactions, Assemblies, rue de la Géraudière, 44000 Nantes, France \\ ${ }^{\mathrm{c} I N R A}$ - Research Unit : Biopolymers, Interactions, Assemblies, BIA-PEPSI - Rue de la Géraudière - BP \\ 71627, 44316 Nantes, France \\ ${ }^{\mathrm{d}}$ INRA - Research Unit : Biopolymers, Interactions, Assemblies, rue de la Géraudière, BP 71627, 44316 \\ Nantes Cedex 3, France \\ zykwinsk@nantes.inra.fr
}

Cellulose, xyloglucan and pectin are the three major polysaccharides of primary cell walls of higher plants. According to the most cited primary cell wall model [1], xyloglucan binds to the surface of cellulose microfibrils and tether adjacent microfibrils. In this "sticky network" model, pectins are assumed to fill the interstices within the xyloglucan/cellulose network [2]. Because of its structural heterogeneity and favorable spatial location within cell wall, pectin seems to be a good candidate for cell wall upholding and expansion [2]. The aim of this work is to study the potential interactions between pectin and cellulose. The first step of the study consisted on isolating cellulose and pectic structural domains from the cell wall. Their assembly was studied using a multidisciplinary in vitro approach getting together binding assays and induced precipitation experiments. It came out that the isolated pectic neutral sugar arabinan and galactan side chains bind in vitro to cellulose microfibrils, contrary to isolated pectic backbone domains of homo- and rhamnogalacturonan I. The hypothesis of non-covalent interactions between pectins, particularly their neutral sugar side chains, and cellulose was therefore raised. Induced precipitation experiments of oriented growth of isolated pectic domains on cellulose microfibrils were then performed. Electron microscopy was applied to investigate the possibility of "shish-kebab" morphology formation [3]. Isolated pectic side chains precipitated attached to the cellulose microfibril surfaces, whereas pectic backbone domains crystallised separately from cellulose microfibrils.

[1] Carpita, N. C. and Gibeaut, D. M. (1993). Structural models of primary cell walls in flowering plants: consistency of molecular structure with the physical properties of walls during growth. Plant J., 3, 1-30 [2] Cosgrove, D. J. (2000). Expensive growth of plant cell walls. Plant Physiol. Biochem., 38, 109-124 [3] Helbert, W. and Chanzy, H. (1994). Oriented growth of V amylose n-butanol crystals on cellulose. Carbohydr. Polym., $24,119-122$ 